\title{
The Furuta Inequality and an Operator Equation for Linear Operators
}

By

\author{
Chia-Shiang LIN *
}

\begin{abstract}
We show that a special form of the Furuta inequality is equivalent to an operator equation $H^{\frac{p-2 r n}{2(n+1)}} T\left(H^{\frac{p+2 r}{n+1}} T\right)^{n} H^{\frac{p-2 r n}{2(n+1)}}=K^{p}$. This result also generalize Lemma 1 in [3] which is about the operator equation $T\left(H^{1 / n} T\right)^{n}=K$. A new characterization of the Löwner-Heinz formula and some applications are given.
\end{abstract}

\section{§1. Notation and Introduction}

Throughout this note the capital letters mean bounded linear operators on a Hilbert space H. $T$ is positive (written $T \geq 0$ ) in case $(T x, x) \geq 0$ for all $x \in H$. If $S$ and $T$ are Hermitian, we write $T \geq S$ in case $T-S \geq O$. I will denote the identity operator. Pedersen and Takesaki [6] proved that if $H, K \geq O$ and $H$ is nonsingular, then $\left(H^{1 / 2} K H^{1 / 2}\right)^{1 / 2} \leq a H$ holds for some $a>0$, if and only if there exists a unique $T \geq O$ such that $T H T=K$. Nakamoto [5] showed the necessary condition by using Douglas's majorization theorem [1], and it turned out to be a very simple proof. Furuta [3] extended and characterized the operator equation to the equation $T\left(H^{1 / n} T\right)^{n}=K$ for any natural number $n$. In this paper we shall use the remarkable Furuta inequality [2] to give a further generalization (as the equation in abstract), which is also a new characterization of a special form of the Furuta inequality. Consequently, a new characterization of the LöwnerHeinz formula and some applications are given.

We recall the following two celebrated results. Firstly, the Douglas theorem [1], i.e., the inequality $A A^{*} \leq \lambda^{2} B B^{*}$ holds for some $\lambda \geq 0$, if and only if there exists a $C$ so that $A=B C$. Moreover, if these statements are valid, then there exists a unique $C$ so that $\|C\| \leq \lambda$. Secondly, the Furuta inequality [2], i.e., if

Communicated by T. Kawai, August 11, 1998. Revised December 1, 1998.

1991 Mathematics Subject Classification: 47B15.

*Deparment of Mathematics, Bishop's Universty, Lennoxville, P. Q. J1M1Z7, Canada.

e-mail: plin@ubishops.ca 
$A \geq B \geq O$, then both inequalities $A^{\frac{p+2 r}{q}} \geq\left(A^{r} B^{p} A^{r}\right)^{1 / q}$ and $\left(B^{r} A^{p} B^{r}\right)^{1 / q} \geq$ $B^{\frac{p+2 r}{q}}$ hold for $p, r \geq 0$, and $q \geq 1$ such that $(1+2 r) q \geq p+2 r$.

Remark that the conditions on $p, r, q$, and the expression $(1+2 r)$ $q \geq p+2 r$ are the best possible with respect to the Furuta inequality [7] (See Figure). More precisely, for $p$, $r \geq 0$, if $q \in(0,1)$ or $(1+2 r) q<p+$ $2 r$, then there are operators $A, B$ : $\mathbb{R}^{2} \rightarrow \mathbb{R}^{2}$ with $A \geq B \geq O$, but $A^{\frac{p+2 r}{q}} \not$ $\left(A^{r} B^{p} A^{r}\right)^{1 / q}$. The above two results have a beautiful relationship as we

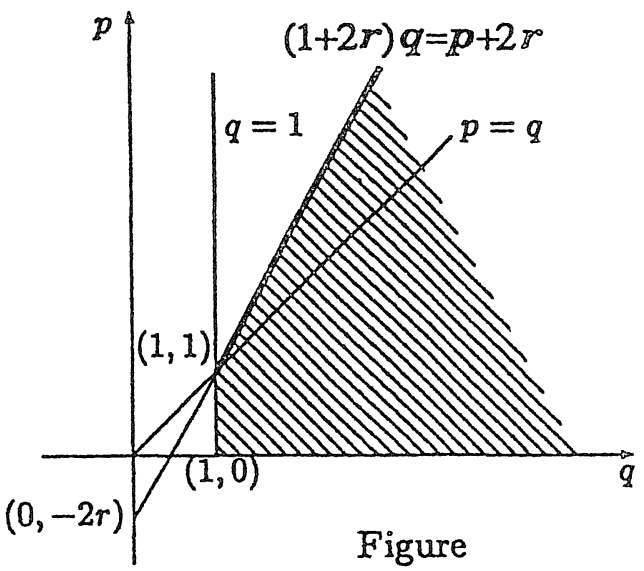
will see in the proof of Theorem below.

\section{§2. Main Result}

We shall make frequent use of the Löwner-Heinz formula throughout the paper, viz. $A^{\alpha} \geq B^{\alpha}$ if $A \geq B \geq O$ for $\alpha \in[0,1]$. If $q$ in the Furuta inequality is a natural number instead, then the inequality may be characterized in terms of an operator equation. We now proceed to derive the main result.

Theorem 1. Let $H \geq K \geq O$, and assume that $H$ is nonsigular. Then the following are equivalent for $p, r \geq 0$, and an integer $n \geq 0$ with $(1+2 r)(n+1) \geq p$ $+2 r$.

(1) $H^{\frac{p+2 r}{n+1}} \geq\left(H^{r} K^{p} H^{r}\right)^{\frac{1}{n+1}} \quad$ (Furuta inequality);

(2) There exists a unique operator $T \geq 0$ with $\|T\| \leq 1$ such that

$$
K^{p}=H^{\frac{p-2 r n}{2(n+1)}} T\left(H^{\frac{p+2 r}{n+1}} T\right)^{n} H^{\frac{p-2 r n}{2(n+1)}} .
$$

Proof. (1) implies (2). As both sides of (1) are positive, and also by Douglas's theorem the inequality (1) implies that there exists a unique $S$ with $\|S\| \leq 1$ such that

$$
\left(H^{r} K^{p} H^{r}\right)^{\frac{1}{2(n+1)}}=H^{\frac{p+2 r}{2(n+1)}} S=S^{*} H^{\frac{p+2 r}{2(n+1)}} .
$$

If we put $T=S S^{*}$, then

$$
\left(H^{r} K^{p} H^{r}\right)^{\frac{1}{n+1}}=H^{\frac{p+2 r}{2(n+1)}} T H^{\frac{p+2 r}{2(n+1)}} .
$$

It follows that 


$$
H^{r} K^{p} H^{r}=\left(H^{\frac{p+2 r}{2(n+1)}} T H^{\frac{p+2 r}{2(n+1)}}\right)^{n+1}=H^{\frac{p+2 r}{2(n+1)}} T\left(H^{\frac{p+2 r}{n+1}} T\right)^{n} H^{\frac{p+2 r}{2(n+1)}}
$$

As $H$ is nonsingular we obtain the required equality in (2).

To show the uniqueness of $T$, for some $Z$ assume $H^{\frac{p-2 r n}{2(n+1)}} T\left(H^{\frac{p+2 r}{n+1}} T\right)^{n} H^{\frac{p-2 r n}{2(n+1)}}$ $=H^{\frac{p-2 r n}{2(n+1)}} Z\left(H^{\frac{p+2 r}{n+1}} Z\right)^{n} H^{\frac{p-2 r n}{2(n+1)}}$, then $T\left(H^{\frac{p+2 r}{n+1}} T\right)^{n}=Z\left(H^{\frac{p+2 r}{n+1}} Z\right)^{n}$, and

$$
\begin{aligned}
& \left(H^{\frac{p+2 r n}{2(n+1)}} T H^{\frac{p+2 r}{2(n+1)}}\right)^{n+1}=H^{\frac{p+2 r}{2(n+1)}} T\left(H^{\frac{p+2 r}{n+1}} T\right)^{n} H^{\frac{p+2 r}{2(n+1)}} \\
& \quad=H^{\frac{p+2 r}{2(n+1)}} Z\left(H^{\frac{p+2 r}{n+1}} Z\right)^{n} H^{\frac{p+2 r}{2(n+1)}}=\left(H^{\frac{p+2 r}{2(n+1)}} Z H^{\frac{p+2 r}{2(n+1)}}\right)^{n+1},
\end{aligned}
$$

and the nonsingularity of $H$ yields $Z=T$. And $\|T\|=\left\|S S^{*}\right\|=\|S\|^{2} \leq 1$.

(2) implies (1). $\left(H^{r} K^{p} H^{r}\right)^{\frac{1}{n+1}}$

$$
\begin{aligned}
& =\left[H^{r} H^{\frac{p-2 r n}{2(n+1)}} T\left(H^{\frac{p+2 r}{n+1}} T\right)^{n} H^{\frac{p-2 r n}{2(n+1)}} H^{r}\right]^{\frac{1}{n+1}} \\
& =H^{\frac{p+2 r}{2(n+1)}} T H^{\frac{p+2 r}{2(n+1)}} \\
& \leq H^{\frac{p+2 r}{n+1}}
\end{aligned}
$$

since $T \leq\|T\| I \leq I$, and $H$ is nonsingular.

Q.E.D.

It was proved in $\left[3\right.$, Lemma 1] that $a H^{1 / n} \geq\left(H^{1 / 2 n} K H^{1 / 2 n}\right)^{\frac{1}{n+1}}$ holds for some $a \geq 0$, if and only if there exists a unique $T \geq O$ such that $T\left(H^{1 / n} T\right)^{n}=K$. This is indeed a special case of our Theorem 1 if $a=1$, in which $p=1, r=\frac{1}{2 n}$, and a natural number $n \geq 1$. Notice that in the proof of Theorem 1 the hypothesis that $H \geq K$ was not used, but it is made only to ensure the validity of the inequality (1) under imposed conditions on $p, r$, and $n$. In fact, all we need is the condition that $H, K \geq O$.

\section{§3. Applications}

The next result is a new characterization of the Löwner-Heinz formula, and the proof is trivial; let $n=r=0$ in Theorem 1 .

Corollary 1. Let $H \geq K \geq O, p \in[0,1]$, and assume that $H$ is nonsingular. Then the following are equivalent.

(1) $H^{p} \geq K^{p} \quad$ (Löwner-Heinz formula);

(2) There exists a unique operator $T \geq 0$ with $\|T\| \leq 1$ such that $K^{p}=H^{p / 2} T H^{p / 2}$.

Recall that $T$ is a $p$-hyponormal operator for $0<p \leq 1$ if $\left(T^{*} T\right)^{p} \geq\left(T T^{*}\right)^{p}$, and it is hyponormal when $p=1$. It is easily seen that $T$ is $p$-hyponormal, if and 
only if $\left|T^{*}\right|^{2 p} \leq|T|^{2 p}$. We write $T=U|T|$ the polar decomposition of $T$ with $U$ the partial isometry, and $|T|$ the positive square root of the positive operator $T^{*} T$. The next result shows some properties of such operator.

Corollary 2. Let $T=U|T|$ be $p$-hyponormal for $0<p \leq 1$. Then, for $q, r \geq 0$, and a natural number $n$ with $(1+2 r)(n+1) \geq q+2 r$, we have

(1) $|T|^{\frac{2 p(q+2 r)}{n+1}} \geq\left(|T|^{2 p r}\left|T^{*}\right|^{2 p q}|T|^{2 p r}\right)^{\frac{1}{n+1}}$;

(2) There exists a unique operator $S \geq 0$ with $\|S\| \leq 1$ such that

$$
\left|T^{*}\right|^{2 p q}=|T|^{\frac{2 q(q-2 r n)}{2(n+1)}} S\left(|T|^{\frac{2 p(q+2 r)}{n+1}} S\right)^{n}|T|^{\frac{2 p(q-2 r n)}{2(n+1)}} .
$$

Moreover, the above two statements are equivalent.

Proof. Since $T$ is $p$-hyponormal let $H=|T|^{2 p}$ and $K=\left|T^{*}\right|^{2 p}$ in Theorem 1 so that $H \geq K \geq O$. We may assume without loss of generality that $|T|$ is nonsingular.

Corollary 3. Let $H, K \geq O, H$ be nonsingular, and $p, r \geq 0$, and let $n$ be a natural number with $(1+2 r)(n+1) \geq p+2 r$. Then,

(1) if there exists a $T \geq O$ such that $K^{p}=H^{\frac{p-2 r n}{2(n+1)}} T\left(H^{\frac{p+2 r}{n+1}} T\right)^{n} H^{\frac{p-2 r n}{2(n+1)}}$, then, for any natural number $m \geq n$, there exists a unique $T^{\prime} \geq O$ such that $K^{p}=$ $H^{\frac{p-2 r m}{2(m+1)}} T^{\prime}\left(H^{\frac{p+2 r}{m+1}} T^{\prime}\right)^{m} H^{\frac{p-2 r m}{2(m+1)}}$

(2) in the statement (1) if $n>m$ instead, then in general there does not exist a $T^{\prime} \geq O$ such that $K^{p}=H^{\frac{p-2 r m}{2(m+1)}} T^{\prime}\left(H^{\frac{p+2 r}{m+1}} T^{\prime}\right)^{m} H^{\frac{p-2 r m}{2(m+1)}}$.

Proof. (1) The given equality implies the relation $H^{\frac{p+2 r}{n+1}} \geq\left(H^{r} K^{p} H^{r}\right) \frac{1}{n+1}$ by Theorem 1 . Since $m \geq n$, the inequality

$$
H^{\frac{p+2 r}{m+1}} \geq\left(H^{r} K^{p} H^{r}\right)^{\frac{1}{m+1}}
$$

holds by the Löwner-Heinz formula, and the conclusion is due to Theorem 1, again.

(2) Since $H^{\frac{p+2 r}{n+1}} \geq\left(H^{r} K^{p} H^{r}\right)^{\frac{1}{n+1}} \geq O$, in view of the Furuta inequality the relation

$$
H^{\frac{p+2 r}{n+1} \cdot \frac{a+2 c}{b}} \geq\left[H^{\frac{(p+2 r) c}{n+1}}\left(H^{r} K^{p} H^{r}\right)^{\frac{a}{n+1}} H^{\frac{(p+2 r) c}{n+1}}\right]^{\frac{1}{b}}
$$

holds for $a, c \geq 0, b \geq 1$ with $(1+2 c) b \geq a+2 c$. Put $a=n+1, b=m+1$, and $c=0$. Then

$$
H^{\frac{p+2 r}{m+1}} \geq\left(H^{r} K^{p} H^{r}\right)^{\frac{1}{m+1}}
$$


but in this case $(1+2 c) b<a+2 c$ as $n>m$. By the best possibility argument mentioned before the above inequality does not exists in general. Consequently, by Theorem 1 there does not exist a $T^{\prime} \geq O$ in general such that $K^{p}=H^{\frac{p-2 r m}{2(m+1)}}$ $T^{\prime}\left(H^{\frac{p+2 r}{m+1}} T^{\prime}\right)^{m} H^{\frac{p-2 r m}{2(m+1)}}$.

Q.E.D.

Remark. It should be pointed out at this stage that if there exists a $T \geq 0$ such that $T\left(H^{1 / n} T\right)^{n}=K$ for some natural number $n$, then for any natural number $m \leq n$, there exists a unique $T^{\prime}$ such that $T^{\prime}\left(H^{1 / m} T^{\prime}\right)^{m}=K$ [3]. However, if $m>n$ instead, then in general there does not exists such $T^{\prime}$ satisfying the equation [4]. It may be of interest to compare opposite properties in the above statement and Corollary 3.

Finally, we may use the second inequality of Furuta to produce a result which is similar to Theorem 1 . Notice that all conditions are exactly the same as in Theorem 1, except assuming nonsingularity of both $H$ and $K$. We shall omit the proof since it may be carried out as in the case of Theorem 1.

Theorem 2. Let $H \geq K \geq O$, and both $H$ and $K$ be nonsingular. Then the following are equivalent for $p, r \geq 0$, and a natural number $n$ with $(1+2 r)(n+$ 1) $\geq p+2 r$.

(1) $\left(K^{r} H^{p} K^{r}\right)^{\frac{1}{n+1}} \geq K^{\frac{p+2 r}{n+1}} \quad$ (Furuta inequality);

(2) There exists a unique operator $T \geq O$ with $\|T\| \leq 1$ such that

$$
K^{p+2 r}=\left(K^{r} H^{p} K^{r}\right)^{\frac{1}{2(n+1)}} T\left[\left(K^{r} H^{p} K^{r}\right)^{\frac{1}{(n+1)}} T\right]^{n}\left(K^{r} H^{p} K^{r}\right)^{\frac{1}{2(n+1)}} .
$$

\section{References}

[1] Douglas, R. G., On majorization, factorization, and range inclusion of operators on Hilbert space, Proc. Amer. Math. Soc., 17 (1966), 413-415.

[2] Furuta, T., $A \geq B \geq O$ assures $\left(B^{r} A^{p} B^{r}\right)^{1 / q} \geq B^{(p+2 r) / q}$ for $r \geq 0, p \geq 0, q \geq 1$ with $(1+2 r) q \geq p+$ 2r, Proc. Amer. Math. Soc., 101 (1987), 85-88.

[3] The operator equation $T\left(H^{1 / n} T\right)^{n}=K$, Linear Algebra Appl.. 109 (1988), 149-152.

[4] Bach, E. and Furuta, T., Counterexample to a question on the operator equation $T\left(H^{1 / n} T\right)^{n}=$ K, Linear Algebra Appl., 177 (1992), 157-162.

[5] Nakamoto, R., On the operator equation $T H T=K$, Math. Japon., 18 (1973), 251-252.

[6] Pedersen, G. K. and Takasaki, M., The operator equation THT=K, Proc. Amer. Math. Soc., 36 (1972), 311-312.

[7] Tanahashi, K., Best possibility of the Furuta inequality, Proc. Amer. Math. Soc., 124 (1996), 141-146. 
